\title{
A Comparison of Various Defogging Techniques
}

\author{
Atul Gujral ${ }^{1}$, Aditi $^{2}$, Shailender Gupta ${ }^{3}$ and Bharat Bhushan ${ }^{4}$ \\ ${ }^{1,2,3,4}$ YMCA University of Science and Technology \\ Faridabad-121006,India \\ 1 atul.gujral5@gmail.com, ${ }^{2}$ aditi22391@gmail.com, \\ ${ }^{3}$ shailender81@gmail.com, ${ }^{4}$ bhrts@yahoo.com
}

\begin{abstract}
This paper compares various defogging techniques such as Dark Channel Prior (DCP), Improved Dark Channel Prior (IDCP), IDCP with guided filter, IDCP with histogram specification, Tarel method, Anisotropic diffusion and Adaptive defogging in HSV color plane. For the purpose of comparison these techniques were implemented on MATLAB-09. The result shows that Adaptive defogging technique has highest value of PSNR among all the techniques having lowest MSE, NCD and MAE value. The computation time for removal of fog is found to follow the trend from low to high is: Anisotropic diffusion, Tarel method, DCP, IDCP, IDCP with guided filter, IDCP with histogram specification and Adaptive defogging.
\end{abstract}

Keywords: Atmospheric light, Atmospheric scattering, Dark channel

\section{Introduction}

Fog, a combination of two components airlight and direct attenuation, degrades the picture quality and creates a lot of problems in video surveillance, tracking and navigation. Light from the atmosphere and light reflected from an object are scattered by these droplets, resulting in the degradation of image quality. Thus it can be said that fog is result of addition of air-light and attenuation to an image, as expressed by the equation (1) given below:

$$
\text { Fog }=\text { Direct Attenuation }+ \text { Air-light }
$$

Attenuation is the gradual loss in intensity of any kind of flux through a medium. Mathematically it can be expressed as:

$$
\text { Direct Attenuation }=\mathrm{J}(\mathrm{x}) \cdot \mathrm{t}(\mathrm{x})
$$

Where $J(x)$ is the Scene Radiance and $t(x)$ is the Medium Transmission. The Direct Attenuation describes the scene radiance and its decay in the medium; it is a multiplicative distortion of the scene radiance. When the atmosphere is homogenous, the transmission $\mathrm{t}(\mathrm{x})$ can be expressed as:

$$
t(x)=e^{-\beta d(x)}
$$

Where $\beta$ is the scattering coefficient of the atmosphere and $d$ is the scene depth of the $x^{\text {th }}$ pixel. The equation (3) indicates that the scene radiance is attenuated exponentially with the depth. If we can recover the transmission, we can also recover the depth up to an unknown scale. 
Air-light is caused due to scattering of light. It adds whiteness to the scene. It is an additive one and is a function of the distance between camera and object. Mathematically the equation of air-light can be described in equation (4) as follows:

$$
\text { Air-light=A }(1-\mathrm{e}-\beta \mathrm{d}(\mathrm{x}))
$$

Where, A is global atmospheric light.

Using equation 1, 2, 3 and 4 the intensity value $\mathrm{I}$ of $\mathrm{x}^{\text {th }}$ pixel a foggy image can be defined mathematically $[1-4]$ by equation 5 :

$$
\mathrm{I}(\mathrm{x})=\mathrm{J}(\mathrm{x}) \cdot \mathrm{t}(\mathrm{x})+\mathrm{A}(1-\mathrm{t}(\mathrm{x}))
$$

In the literature various defogging strategies have been proposed that can be classified as multiple image methods which have Polarization filter as well as single image method which have Dark Channel Prior (DCP), Improved Dark Channel Prior (IDCP), IDCP with guided filter, IDCP with histogram specification, Tarel method, Anisotropic diffusion and Adaptive defogging in HSV colour plane. The rest of the paper is structured as follows: Section 2 describes various defogging algorithms, Section 3 describes simulation setup parameters and tool used, Section 4 describes performance metrics and Section 5 shows the results of various algorithms followed by analysis and concluding remarks.

\section{Literature Survey of Defogging Strategies:}

A Various defogging strategies used in this paper are shown in Figure 1:

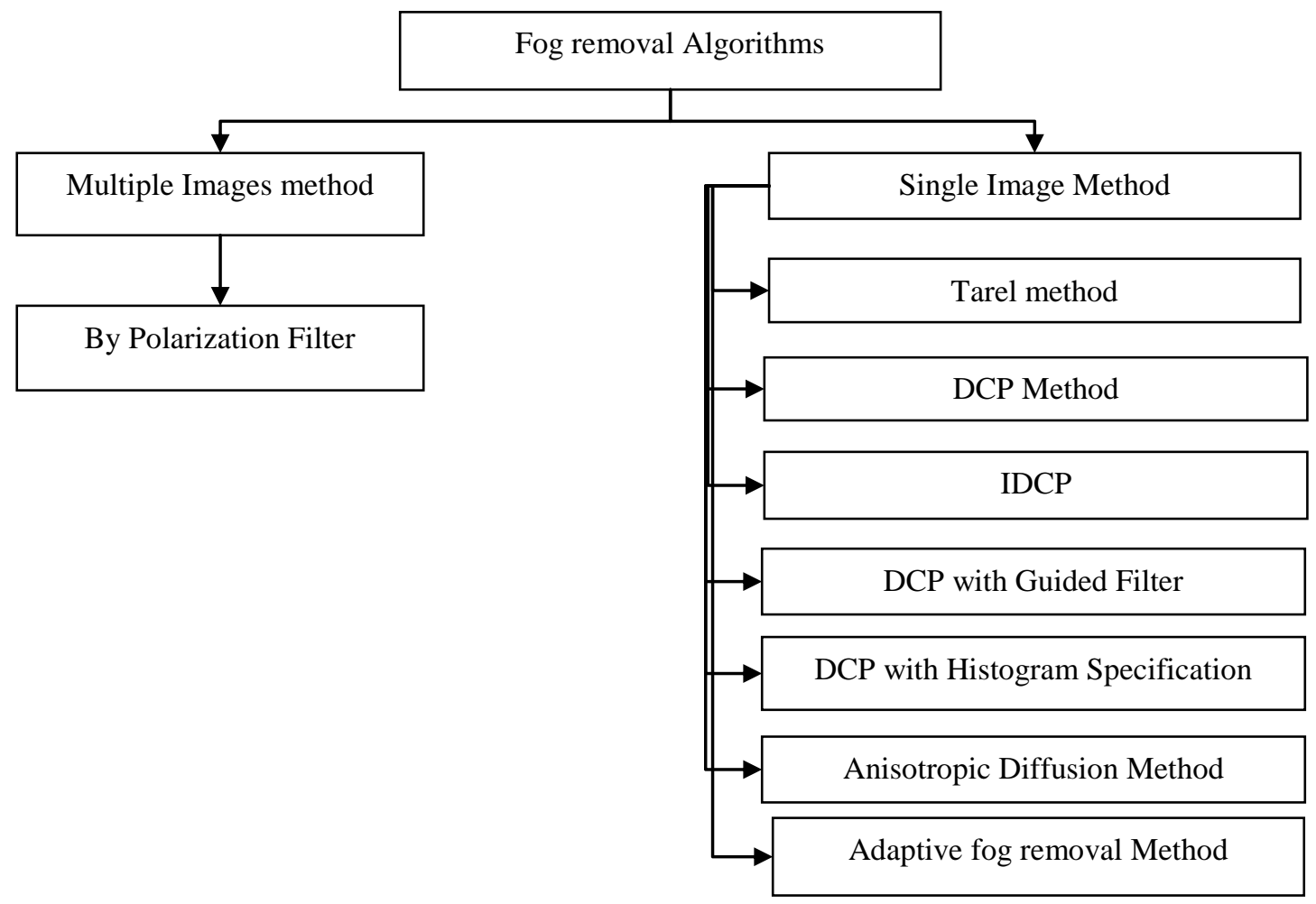

Figure 1. Various Fog Removal Techniques 


\subsection{Removal of Fog by Polarization Filters:}

2.1.1. Fog Removal Process: Fog from the image can be removed by multiple image method by using polarization filter [5-6]. While capturing an image from a distance $\mathrm{z}$, the reflected light from the object to the camera is called direct light, D. The portion of light scattered due to environmental illumination in the same direction of direct light arriving in the camera is termed as airlight, A. In Figure 2 the algorithm of defogging using polarization filter is shown.

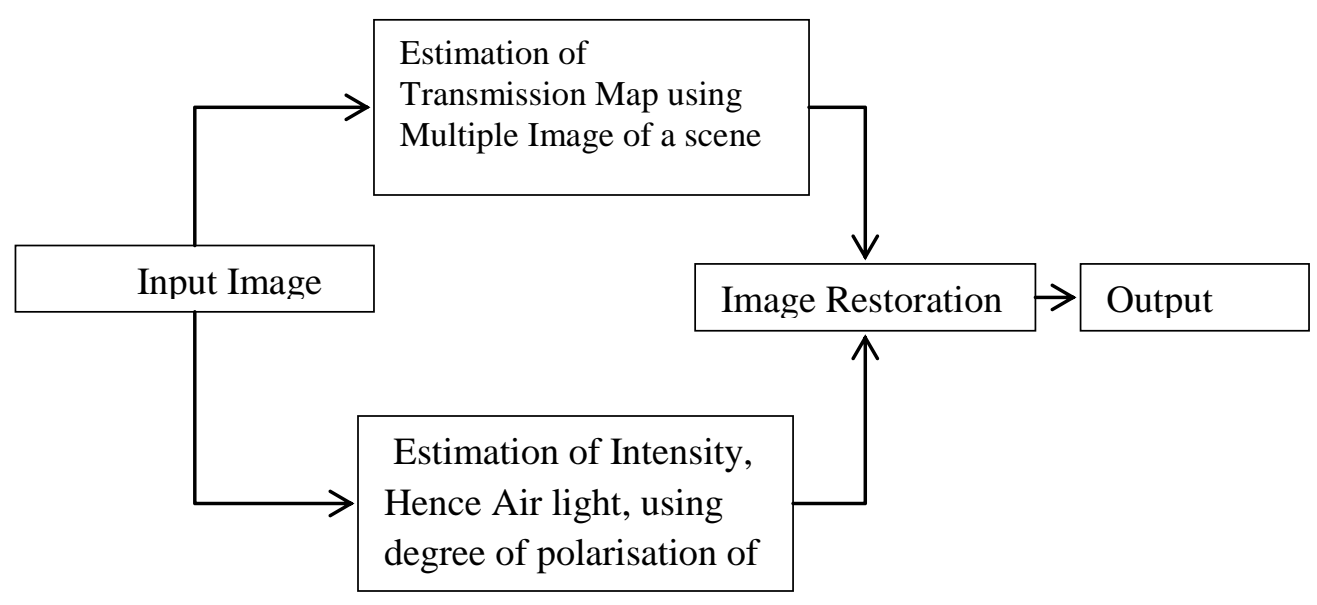

Figure 2. Block Diagram of Defogging by Polarization Filter

Assume that the direct light may be denoted by equation (6):

$$
\mathrm{D}=\mathrm{J}(\mathrm{x}) \cdot \mathrm{t}(\mathrm{x})
$$

Where $\mathrm{x}$ is the real world ray corresponding to the pixel and $\mathrm{J}(\mathrm{x})$ is the surface radiance vector and $\mathrm{t}(\mathrm{x})$ as defined by equation (7):

$$
\mathrm{t}=\exp \left[-\int_{0}^{\mathrm{z}} \beta\left(\mathrm{z}^{\prime}, \lambda\right) \mathrm{dz} \mathrm{z}^{\prime}\right]
$$

$t(x)$ is the transmittance of the atmosphere. It depends on the distance $\mathrm{z}$ between the object and the camera and on the atmospheric coefficient $\beta$. When atmospheric attenuation coefficient is independent to the position, $\beta(z$ ') is the constant and then $t$ can be written as :

$$
\mathrm{t}=\exp \left[-\int_{0}^{\mathrm{z}} \beta \mathrm{dz} \mathrm{z}^{\prime}\right]=\exp \left[-\beta \int_{0}^{\mathrm{z}} \mathrm{dz} \mathrm{z}^{\prime}\right]=\exp [-\beta \mathrm{z}]
$$

The airlight may be described as the light coming from the source towards the observer. When we use polarization filters, we consider that the airlight is partially polarized. Hence, the polarizer may be modulated to estimate the airlight components. When the polarizer is rotated, we obtain two states where the intensity will be either minimum or maximum. This intensity changes due to the reason that another direction of airlight is filtrated. The orientation where the airlight contribution is most intense, it is denoted by $\mathrm{A}(\mathrm{x}) \max$ and where the airlight contribution is least, it is denoted by $\mathrm{A}(\mathrm{x})^{\mathrm{min}}$.

Hence, the airlight $\left(\mathrm{A}_{\text {light }}\right)$ may be expressed by equation (9): 


$$
\mathrm{A}_{\text {light }}=\mathrm{A}(\mathrm{x})^{\max }+\mathrm{A}(\mathrm{x})^{\text {min }}
$$

Here, the assumption is that the direct transmission is not polarized; its energy is evenly distributed between the polarization components. Hence, the intensity of scene can be expressed by equation (10) and (11):

$$
\begin{gathered}
\mathrm{I}(\mathrm{x})^{\min }=\mathrm{D}(\mathrm{x}) / 2+\mathrm{A}(\mathrm{x})^{\min } \\
\mathrm{I}(\mathrm{x})^{\max }=\mathrm{D}(\mathrm{x}) / 2+\mathrm{A}(\mathrm{x})^{\max }
\end{gathered}
$$

Where $\mathrm{D}(\mathrm{x})$ is the direct attenuation.

We use the definition of visibility to define the degree of polarization $\mathrm{p}$, which defines the airlight degree of polarization as:

$$
\mathrm{p}(\mathrm{x})=\left(\mathrm{A}(\mathrm{x})^{\max }-\mathrm{A}(\mathrm{x})^{\mathrm{min}}\right) / \mathrm{A}_{\text {light }}
$$

Supposition is that $\mathrm{p}$ is a constant, ranging from 0 to 1 .

Equation (12) defines the degree of polarization $p$ for airlight component as:

$$
\begin{aligned}
& \mathrm{A}(\mathrm{x})^{\mathrm{max}}=\mathrm{A}_{\text {light }}(1-\mathrm{p}) / 2 \\
& \mathrm{~A}(\mathrm{x})^{\mathrm{min}}=\mathrm{A}_{\text {light }}(1+\mathrm{p}) / 2
\end{aligned}
$$

The total intensity can be expressed from equation (13) and (14) as:

$$
\mathrm{I}(\mathrm{x})^{\mathrm{total}}=\mathrm{I}(\mathrm{x})^{\mathrm{max}}+\mathrm{I}(\mathrm{x})^{\min }
$$

2.1.2. Image Recovery: For recovering an image $(\mathrm{J}(\mathrm{x}))$, the airlight must be estimated given by equation (16) below:

$$
\mathrm{A}^{\prime}{ }_{\text {light }}=\left(\mathrm{I}(\mathrm{x})^{\max }-\mathrm{I}(\mathrm{x})^{\mathrm{min}}\right) / \mathrm{p}
$$

Finally, the image is recovered using the airlight and transmittance as in equation (17):

$$
\mathrm{J}^{\prime}(\mathrm{x})=\left(\mathrm{I}(\mathrm{x})^{\text {total }}-\mathrm{A}^{\prime}{ }_{\text {light }}\right) / \mathrm{t}^{\prime}(\mathrm{x})
$$

\subsection{Dark Channel Prior Technique}

This method [7], [19] estimates transmission map and air-light to recover original image from foggy image. To estimates the transmission map, it uses the lowest intensity pixel of image in 3 colour planes in patch sizes of different values. Block diagram for DCP algorithm is shown in Figure 3.

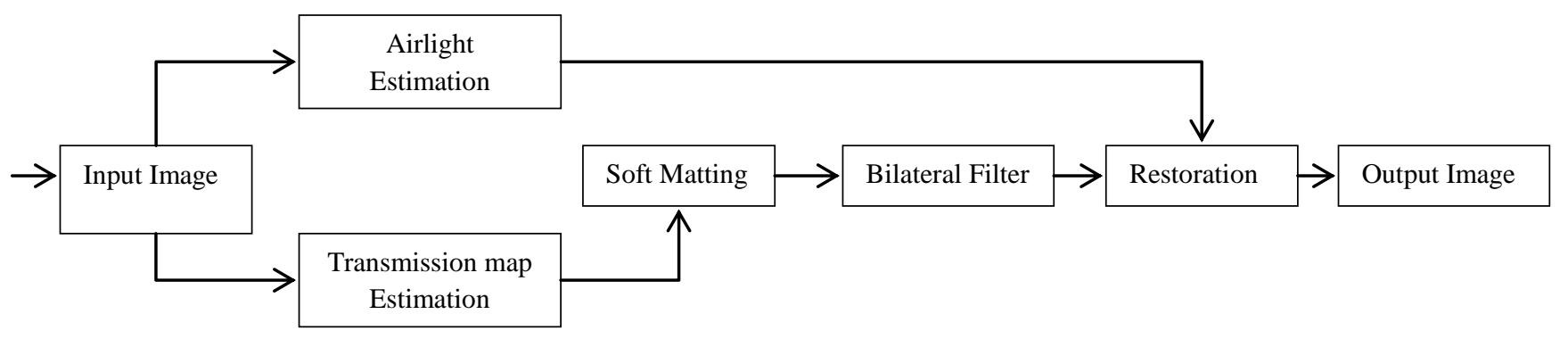

Figure 3. Block Diagram of DCP Algorithm 
To understand the process completely, detailed explanation of each step of algorithm is given as follows:

2.2.1. Transmission Map Estimation: First, normalize the image haze equation given in equation (5):

$$
\frac{\mathrm{I}^{\mathrm{C}}(\mathrm{x})}{\mathrm{A}^{\mathrm{c}}}=\frac{\mathrm{t}(\mathrm{x}) \mathrm{Jc}(\mathrm{x})}{\mathrm{A}^{\mathrm{c}}}+(1-\mathrm{t}(\mathrm{x}))
$$

Where $\mathrm{I}^{\mathrm{c}}$ is foggy image intensity of the $\mathrm{x}^{\text {th }}$ pixel and $\mathrm{t}$ is its transmission map. Take local patch $\Omega(\mathrm{x})$ as constant and transmission map as t', then above equation can be written as:

$$
\operatorname{miny} € \Omega(x)\left(\operatorname{minc}\left(\frac{1^{\mathrm{C}}(\mathrm{x})}{\text { sf }_{\mathrm{f}}}\right)\right)=\mathrm{t}^{\prime}(\mathrm{x}) \operatorname{miny} € \Omega(\mathrm{x})\left(\operatorname{minc}\left(\frac{\mathrm{\rho}^{\mathrm{C}}(\mathrm{x})}{\mathrm{s}_{\mathrm{f}}}\right)\right)+\left(1-\mathrm{t}^{\prime}(\mathrm{x})\right)
$$

Since $\mathrm{t}^{\prime}(\mathrm{x})$ is a constant in the patch. As the scene radiance $\mathrm{J}$ is a haze-free image, the dark channel of $\mathrm{J}$ is close to zero due to the dark channel prior:

$$
\mathrm{J}(\mathrm{x})=0
$$

Then equation (19) can be rewritten as:

$$
\mathrm{t}^{\prime}(\mathrm{x})=1-\min _{\mathrm{y} \in \Omega(\mathrm{x})}\left(\min _{\mathrm{c}}\left(\frac{\mathbb{1}^{\mathrm{c}}(\mathrm{x})}{A^{\mathrm{E}}}\right)\right)
$$

Due to aerial perspective, modified transmission map [4], [8] is shown in equation (22):

$$
\mathrm{t}^{\prime}(\mathrm{x})=1-\mathrm{w}^{*} \min _{\mathrm{y} \in \Omega(\mathrm{x})}\left(\min _{\mathrm{c}}\left(\frac{\mathrm{I}^{\mathrm{C}}(\mathrm{x})}{\mathrm{A}^{\mathrm{c}}}\right)\right)
$$

where $0<\mathrm{w}<1$

$\mathrm{w}$ is taken as 0.95 .

In the next sections, atmospheric light estimation and soft matting method is proposed to refine the transmission map [9].

2.2.2. Atmospheric Light Estimation: We pick the top 0.1 percent brightest pixels in the dark channel as given by Tan et. al., [4].

2.2.3. Soft Matting: Refined transmission map is denoted by $t(x)$. Rewriting $t(x)$ and $t^{\prime}(x)$ in their vector forms as $t$ and $t^{\prime}$, we minimize the following cost function as in equation (23):

$$
\mathrm{E}(\mathrm{y})=\mathrm{t}^{\mathrm{T}} \mathrm{Lt}+\lambda\left(\mathrm{t}-\mathrm{t}^{\mathrm{T}}\right)^{\mathrm{T}}\left(\mathrm{t}-\mathrm{t}^{\mathrm{\prime}}\right) .
$$

The first term is a smoothness term and the second term is a data term with a weight $\lambda$. The matrix L is called the matting Laplacian matrix [9] defined as:

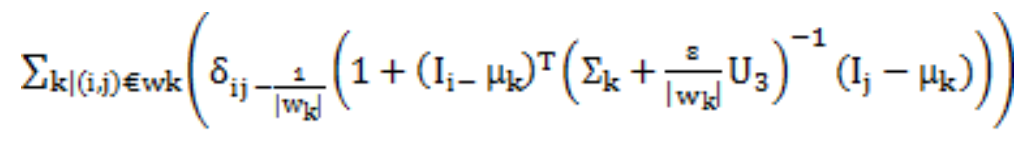

$I_{i}$ and $I_{j}$ are the colors of the input image I at pixels I and $j, \delta_{i j}$ is the Kronecker delta, $\mu_{k}$ and $\Sigma_{\mathrm{k}}$ are the mean and covariance matrix of the colours in window wk, U3 is a 3 X3 identity matrix, $\varepsilon$ is a regularizing parameter, and $\left|\mathrm{w}_{\mathrm{k}}\right|$ is the number of pixels in the window $\mathrm{w}_{\mathrm{k}}$.

The optimal $\mathrm{t}$ can be obtained by solving the following sparse linear system as given by equation (25):

$$
(\mathrm{L}+\lambda \mathrm{U}) \mathrm{t}=\lambda \mathrm{t}^{\prime}
$$


where $U$ is an identity matrix of the same size as $L$. We set the value of $\lambda 10^{-4}$ in the experiments.

In the next step bilateral filter is applied.

2.2.4. Bilateral Filter: The purpose of applying bilateral filter [10] is to smoothen the small scale textures of image.

2.2.5. Image Restoration: In this step, image is restored; the value of restored image is given in equation (26):

$$
J(x)=\frac{I(x)-A}{\max \left(t(x)_{i} t_{0}\right)}+A
$$

A typical value of $t_{0}$ is 0.1 .

This method may not be efficient at times as it may corrupt the image when it contains multiple large lamps since the estimation of atmospheric light is automatic.

\subsection{Improved Single Image Dehazing using Dark Channel Prior}

In this proposal [11], a novel estimation of atmospheric light has been proposed. Compared to the Dark Channel Prior method, it can obtain better results and also resolves that the substantial sky region of recovered image usually tends to be distorted. Figure 4 shows the block diagram of IDCP.

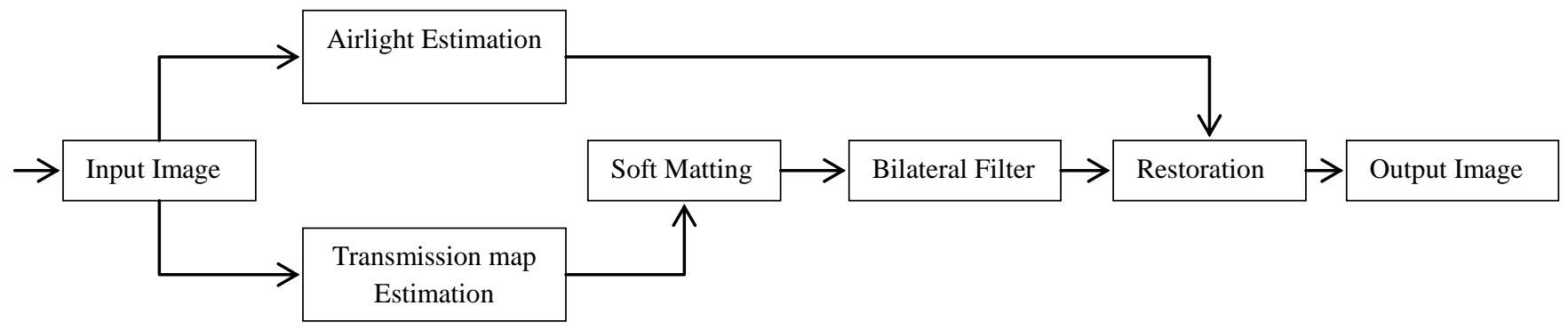

Figure 4. Block Diagram of IDCP Algorithm

To make the process clear, detailed explanation of each step of algorithm is as follows:

2.3.1. Dark Channel Prior: It is based on the observation that in most of the non-sky patches, at least one color channel has very low intensity at some pixels, and the image is defined as:

$$
\mathrm{J}^{\mathrm{dark}}(\mathrm{x})=\min _{\mathrm{c} \in\{\mathrm{r}, \mathrm{g}, \mathrm{b}\}}\left(\min _{\mathrm{y} \in \Omega(\mathrm{x})}\left(\mathrm{J}^{\mathrm{c}}(\mathrm{y})\right)\right)
$$

2.3.2. Atmospheric Light Estimation: If the window size is increased to 31, the atmospheric light will be properly estimated amongst the pixels having thick haze. The group of brightest pixels, belonging to the dark channel, are estimated by the algorithm that finds the max R, G, $\mathrm{B}$ values amongst them to assemble the atmospheric light. It may be chosen by two methods:

1. One can find the region where the scene appears to be farthest from the camera and use a rectangle to select it.

2. Compute local dark channel in the rectangular region. 
2.3.3. Transmission Map Estimation: First, normalize the image haze equation as given in equation (5) and modified equation is given below:

$$
\frac{\mathrm{I}^{\mathrm{C}}(\mathrm{x})}{\mathrm{A}^{\mathrm{c}}}=\frac{\mathrm{t}(\mathrm{x}) \mathrm{Jc}(\mathrm{x})}{\mathrm{A}^{\mathrm{c}}}+(1-\mathrm{t}(\mathrm{x}))
$$

Where $\mathrm{I}^{\mathrm{c}}$ is foggy image intensity of the $\mathrm{x}^{\text {th }}$ pixel and $\mathrm{t}$ is its transmission map. Local patch $\Omega(\mathrm{x})$ is constant and transmission map as t', then equation (28) can be written as:

$$
\operatorname{miny} € \Omega(x)\left(\operatorname{minc}\left(\frac{1^{c}(x)}{A^{c}}\right)\right)=t^{x}(x) \operatorname{miny} € \Omega(x)\left(\operatorname{minc}\left(\frac{\mathrm{I}^{c}(x)}{A^{c}}\right)\right)+\left(1-t^{x}(x)\right)
$$

since $t^{\prime}(x)$ is a constant in the patch. As the scene radiance $\mathrm{J}$ is a haze-free image, the dark channel of $\mathbf{J}$ is close to zero due to the dark channel prior:

$$
\mathrm{J}(\mathrm{x})=0
$$

Then equation (29) can be rewritten as:

$$
\mathrm{t}^{\prime}(\mathrm{x})=1-\min _{\mathrm{c}}\left(\min _{\mathrm{y} \in \Omega(\mathrm{x})}\left(\frac{\mathrm{I}^{\mathrm{c}}(\mathrm{x})}{\mathbf{A}^{\mathrm{c}}}\right)\right)
$$

It directly provides the estimation of the transmission but it contains some block effects since transmission is not always constant in a patch. Hence, a soft matting algorithm is used to refine the transmission in the same manner as described in DCP technique.

2.3.4. Image Restoration: In this step, image is restored. The value of restored image is given by:

$$
\mathrm{J}(\mathrm{x})=\frac{\mathrm{I}(\mathrm{x})-\mathrm{A}}{\operatorname{sen} \mathrm{x}}+\mathrm{A}
$$

A typical value of $\mathrm{t} 0$ is 0.35 .

When $\mathrm{t}_{0}$ is 0.1 , it needs to be increased, when an image contains substantial sky regions, to avoid artefacts. With $\mathrm{t}_{0}$ as 0.35 , the sky region becomes brighter and smoother.

\subsection{Improved Haze Removal Algorithm using Dark Channel Prior (based on Guided Filter)}

Dark Channel Prior may not work on particular images, especially where the large grey region is similar to the global atmospheric light. In this approach, atmospheric light is estimated, based on the imaging law of very dense hazy regions, more accurately. Also, a replacement mechanism is designed for optimizing the rough transmission map, which can weaken treatment in invalid areas. Figure 5 shows the block diagram of defogging process.

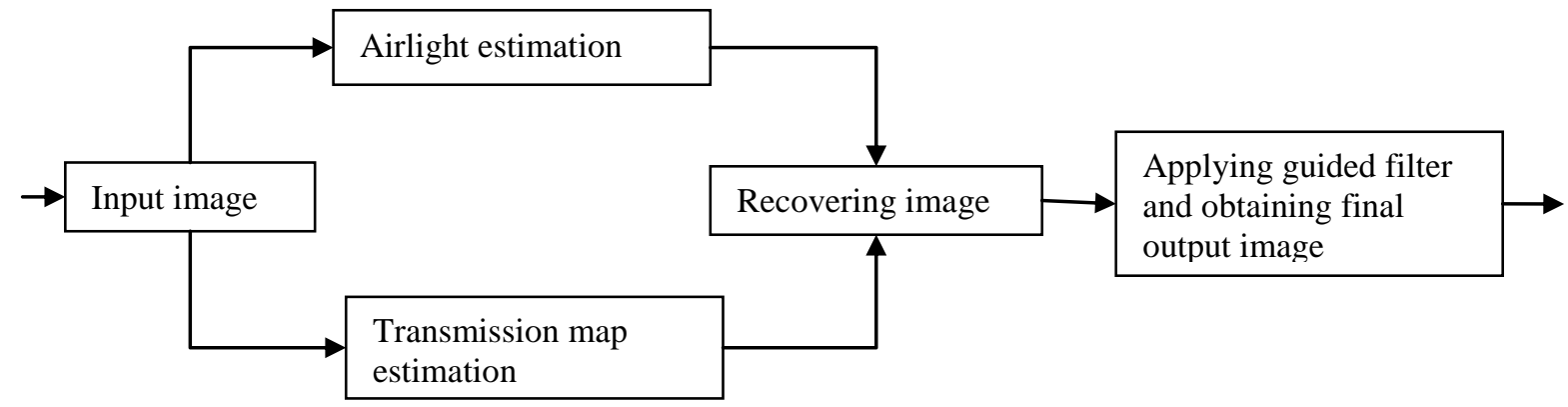

Figure 5. Block Diagram of IDCP Algorithm Using Guided Filter 
The algorithm to implement the IDCP [12] is shown in Figure 6:

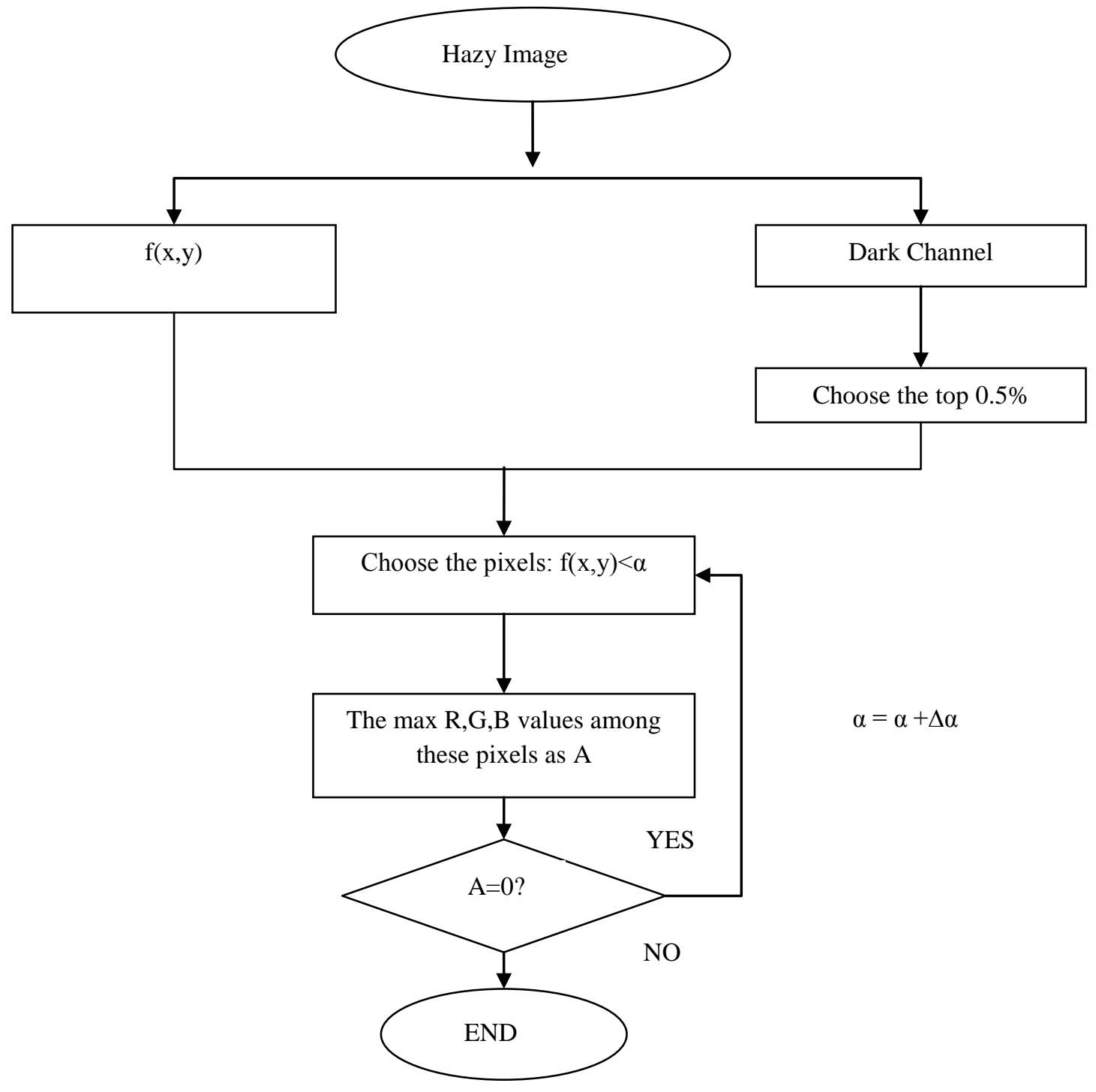

Figure 6. Flowchart of the IDCP Using Guided Filter

2.4.1. Dark Channel Prior: It is based on the observation that in most of the nonsky patches, at least one colour channel has very low intensity at some pixels. The image is defined in equation (33):

$$
\mathrm{J}^{\mathrm{dark}}(\mathrm{x})=\min _{\mathrm{c} \in\{\mathrm{r}, \mathrm{g}, \mathrm{b}\}}\left(\min _{\mathrm{y} \in \Omega(\mathrm{x})}\left(\mathrm{J}^{\mathrm{c}}(\mathrm{y})\right)\right)
$$

Where $\mathrm{J}^{\mathrm{c}}$ is a color channel of $\mathrm{J}$ and $\Omega(\mathrm{x})$ is a local patch centered at $\mathrm{x}$.

2.4.2. Atmospheric Light Estimation: The Dark Channel Prior method cannot ensure whether the atmospheric light belongs to the dense hazy areas or not since the restored image with large gray regions will look dim when the value of A is too large. Hence, the heavy hazy regions are manually segmented in order to analyses these characteristics. To estimate the 
atmospheric light, the inequality in the equation (34) must be satisfied for the pixels of the most haze-opaque region:

$$
\mathrm{f}(\mathrm{x}, \mathrm{y})=\max _{\mathrm{c} \in\{\mathrm{r}, \mathrm{g}, \mathrm{b}\}} \mathrm{I}^{\mathrm{c}}(\mathrm{x}, \mathrm{y})-\min _{\mathrm{c} \in\{\mathrm{r}, \mathrm{g}, \mathrm{b}\}} \mathrm{I}^{\mathrm{c}}(\mathrm{x}, \mathrm{y})<\alpha
$$

where $f$ : deviation map of the hazy image

$\alpha$ : constant parameter ( 0 to 5 ).

2.4.3. Transmission Map Estimation: R, G, B values are almost same and close to the atmospheric light, in case of large grey region images. Hence, there will be a problem of colour distortion in recovered image. To solve this issue, reduction restoration is necessary on such regions. Hence, a replacement mechanism is proposed to optimize the transmission map expressed in equation (35):

$$
\mathrm{t}^{\prime}(\mathrm{x}, \mathrm{y})=\left\{\begin{array}{c}
1, \mathrm{f}(\mathrm{x}, \mathrm{y})<\sigma \text { and } \mathrm{I}(\operatorname{dark})(\mathrm{x}, \mathrm{y})>\mu \\
\operatorname{tbar}(\mathrm{x}, \mathrm{y})
\end{array}\right.
$$

Where ,tbar=1- $\omega \max _{\mathrm{c} \in\{\mathrm{r}, \mathrm{g}, \mathrm{b}\}} \min _{\mathrm{y \in w}(\mathrm{x})}\left(\mathrm{I}^{\mathrm{c}}(\mathrm{x}, \mathrm{y}) / \mathrm{A}^{\mathrm{c}}\right)$

$\omega$ is taken as 0.95 .

The corresponding rough transmission will be replaced by one, when the deviation in the input image is smaller than the threshold value $\sigma$ and the value of the corresponding pixel in the dark channel is larger than judgment threshold $\mu$. Otherwise, the value remains unchanged. Here, $\sigma$ should be set from 4 to 10 while $\mu$ is the segment threshold of the dark channel calculated by OTSU method.

But this transmission needs refinement as it is a fixed value in a patch and hence, contains halo effects in the region of discontinuous depth and block artefacts in the estimated scene radiance. Hence the Guided Filter, which is also an edge-preserving smoothing operator, could eliminate halos effectively.

Filter operation is expressed by equation (37):

$$
\mathrm{t}(\mathrm{i})=1 / \mathrm{N} \sum_{\mathrm{k}=\mathrm{iew}} \mathrm{akli}^{\mathrm{a}}+\mathrm{bk}
$$

where, $a_{k}$ and $b_{k}$ are linear coefficients, determined by the input image and the estimated rough transmission $\mathrm{N}$ is the number of pixels in the window $\mathrm{w}_{\mathrm{k}}$.

2.4.4. Image Restoration: In this step, image is restored. The value of restored image is given by:

$$
\mathrm{J}(\mathrm{x})=\frac{\mathrm{I}(\mathrm{x})-\mathrm{A}}{\max \left(\mathrm{t}(\mathrm{x})_{1} \mathrm{t}_{0}\right)}+\mathrm{A}
$$

A value of t0 ranges from 0.1 to 0.75 in order to make results satisfy the demand of SNR.

\subsection{An Improved Single Image Haze Removal Algorithm Based on Dark Channel Prior and Histogram Specification}

DCP approach is concise and graceful, but still there exist three defects as elaborated:

Firstly, the term (1-t(x)) is equivalent to the thickness of haze; therefore, when different intensity of foreground irradiance passes through same thickness haze, these have the same degree of attenuation reducing the image contrast.

Secondly, the term direct attenuation is not suitable to be presented as linear relation with image intensity and hence DCP method underestimates the attenuation of the foreground irradiance though haze. 
Thirdly, if the haze image has large background area or low contrast, DCP result will cause bad result on dark background and low contrast because it merges the scene with the thick haze.

Hence, if the image has the feature of low contrast and owning large area with background, DCP method will obtain an indistinct and dim result. To overcome these defects, improved DCP with histogram specification [13] has been proposed to improve the contrast of the recovered image which involves rebuilding the histogram of image.

Principle of histogram states that in high intensity regions, there always occur a lot of sharp points. The start point of such sharp point can be utilised as a boundary of foreground and background. Compared with the hazy image, histogram of image, after haze removal, has the tendency of left shifting, i.e., the image after haze removal is darker than the hazy image. Figure 7 shows the flow chart of Histogram specification process.

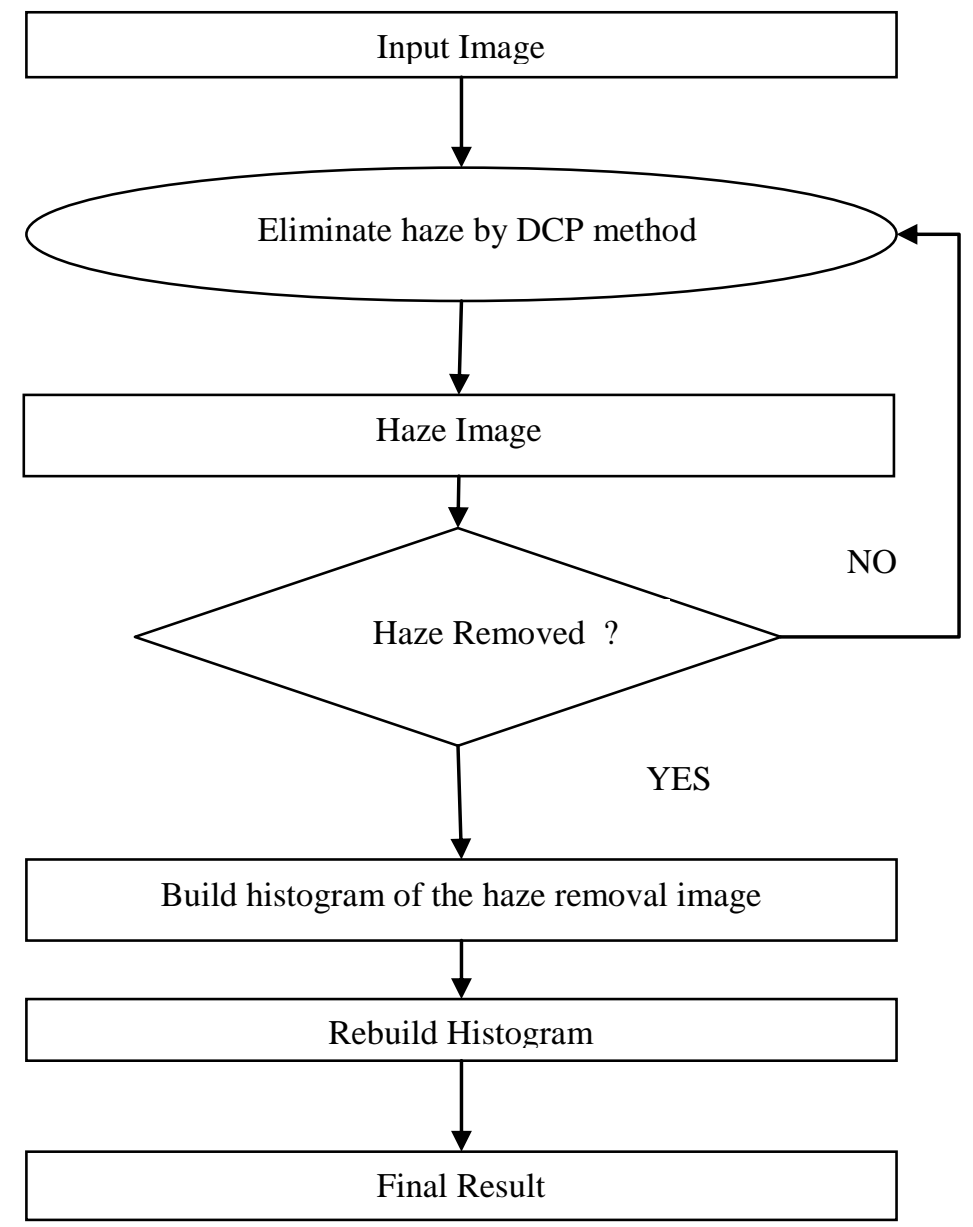

Figure 7. Flowchart of Histogram Specification Method

The detailed algorithm is explained as follows:

1. Build the histogram A of haze image, and find intensity xA corresponding to the peak in the high intensity region of histogram. 
2. Remove the haze in image using DCP method, then build the histogram B of haze removal image, find the start point $\mathrm{xB}$ of the sharp points in histogram $\mathrm{B}$.

3. Divide the histogram $B$ into two parts by the point $x B$. Low intensity part B1 presents foreground distribution and the high intensity part B2 presents background distribution.

4. Rebuild B2. Use a quadratic function to replace $\mathrm{B} 2$ by setting $\mathrm{xA}$ and $\mathrm{xB}$ as start point and end point respectively and the function value is adaptively determined by histogram $\mathrm{B}$.

Combine B1 and new B2 and obtain the rebuilt histogram B'. Do the histogram specification and get the result image.

\subsection{Adaptive Defogging Algorithm}

The method [14] presented here shows an adaptive image defogging algorithm that uses HSV color space model. Figure 8 shows the block diagram. The foggy image equation (39) used in this paper is as follows:

$$
\mathrm{f}^{\mathrm{c}}(\mathrm{x}, \mathrm{y})=\mathrm{f}_{\mathrm{d}}^{\mathrm{c}}(\mathrm{x}, \mathrm{y}) \mathrm{t}(\mathrm{x}, \mathrm{y})+\mathrm{A}(1-\mathrm{t}(\mathrm{x}, \mathrm{y})), \text { for } \mathrm{c}\{\mathrm{R}, \mathrm{G}, \mathrm{B}\}
$$

where first term in RHS is direct attenuation and second term is airlight of a particular pixel. It also involves estimation of air-light and transmission map to refine the foggy image.

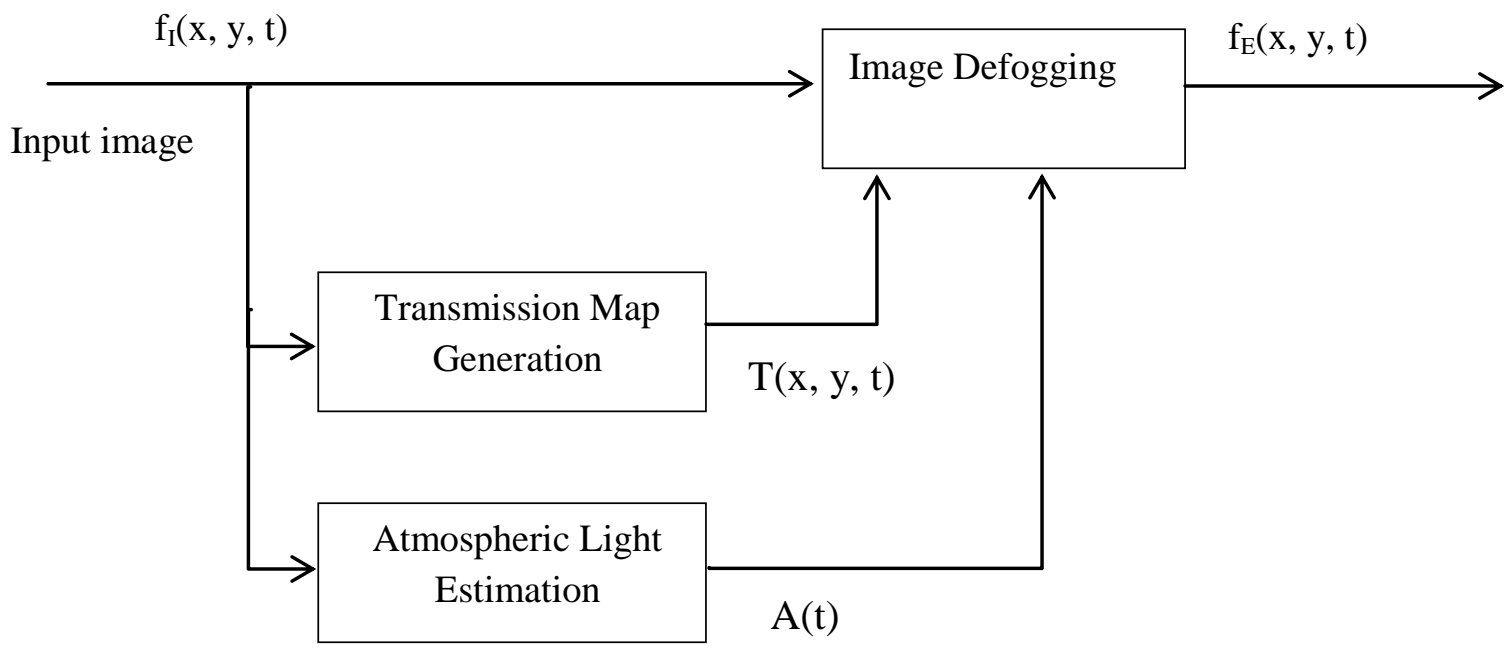

Figure 8. Flowchart of Adaptive Defogging Method

2.6.1. Atmospheric Light Estimation: This method takes highest intensity (V) value in the HSV colour space for the estimation of atmospheric light. It is advantageous from other techniques in the sense that the others [4] take the brightest pixel among all colour channels in the foggy image that may result in the colour distortion problem if originally image contains white objects without fog.

2.6.2. Transmission Map Estimation: It is similar to method used in DCP [7]. In this method for generating the transmission map, search for the lowest intensity in the patch centred at $(\mathrm{x}, \mathrm{y})$, called $\Omega(\mathrm{x}, \mathrm{y})$

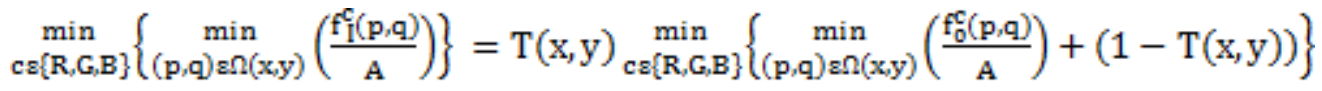


Ideally the minimum intensity in a patch of ideal clear image should be zero, therefore equation (40) becomes:

$$
\mathrm{T}(\mathrm{x}, \mathrm{y})=1-\min _{\operatorname{cs}(\mathrm{R}, \mathrm{G}, \mathrm{B}\}}\left\{\min _{(\mathrm{p}, \mathrm{q}) \mathrm{gn}(\mathrm{x}, \mathrm{y})}\left(\frac{\mathrm{f}_{\mathrm{C}}^{\mathrm{C}}(\mathrm{p}, \mathrm{q})}{\mathrm{A}}\right)\right\}
$$

where $T(x, y)$ is transmission, $f_{I}^{c}$ is foggy image intensity pixel value and $A$ is atmospheric light

2.6.3. Image restoration: Given the atmospheric light $A$ and the modified transmission map $t(x, y)$, the $V$ component of the defogged image frame can be recovered as:

$$
f_{d}^{\mathrm{V}}(\mathrm{x}, \mathrm{y}, \mathrm{c})=\frac{\left(\mathrm{f}^{\mathrm{N}}(\mathrm{x}, \mathrm{y}, \mathrm{c})-\mathrm{A}(\mathrm{t})\right)}{\mathrm{t}(\mathrm{x}, \mathrm{y}, \mathrm{c})}+\mathrm{A}
$$

where $\mathrm{c}$ is frame number.

\subsection{Anisotropic Diffusion Algorithm}

This method is independent of the density of fog and does not require user intervention. It can handle colour as well as grey images. Figure 9 shows the block diagram of defogging algorithm. In addition, this algorithm can work for HSI (hue, saturation and intensity) model which further reduces the computation. It [15] has a wide application in tracking and navigation, consumer electronics and entertainment industries.

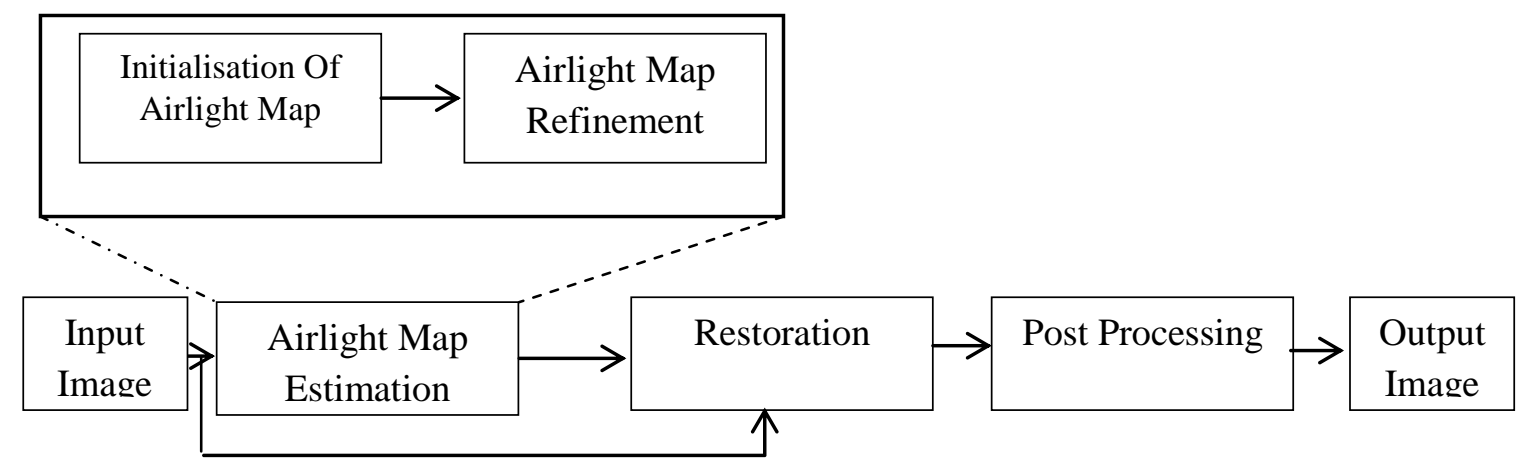

Figure 9. Block Diagram of Anisotropic Diffusion Method

2.7.1. Initial Estimation of Airlight Map: As in DCP algorithm airlight estimation is carried out as follows [7] shown in equation (43):

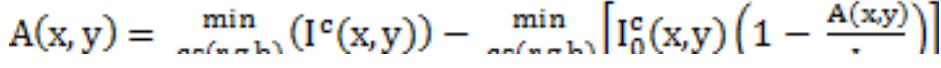

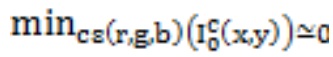

$$
\begin{aligned}
& A_{0(x, y)}=\beta \min _{\operatorname{cr}(r, g b)}\left(I^{c}(x y)\right)
\end{aligned}
$$

Where $\beta$ is a constant. For grey scle image equation (45) becomes:

$$
\left.A_{0}(x, y)=\beta I(x, y)\right)
$$

2.7.2. Refinement: According to perona, malik [16] equation Anisotropic diffusion can be represented as shown in equation (47): 


$$
\frac{\partial \mathrm{A}}{\tau_{\mathrm{s}}}=\operatorname{div}(\alpha(\mathrm{x}, \mathrm{y}, \mathrm{t}) \nabla \mathrm{A})=\alpha(\mathrm{x}, \mathrm{y}, \mathrm{t}) \Delta \mathrm{A}+\nabla \alpha \nabla \mathrm{A}
$$

Where div is divergence operator and $\alpha$ is conduction coefficient. $\nabla$ and $\Delta$ are gradient and Laplacian operators, respectively. If $\mathrm{a}(\mathrm{x}, \mathrm{y}, \mathrm{t})$ is constant over time and space then (47) reduces to isotropic heat diffusion equation.

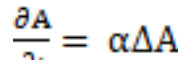

To encourage the smoothing within a region instead of the smoothing across the edges, $\alpha$ should be 1 in the interior region and 0 at the edges. If $\mathrm{E}(\mathrm{x}, \mathrm{y}, \mathrm{t})$ is the estimation of the boundaries, then according to the Perona-Malik equation the conduction coefficient should be chosen in equation (49):

$$
\alpha=\mathrm{g}(\|\mathrm{E}\|)
$$

Here $\mathrm{g}($.$) is :$

$$
\mathrm{g}(\|\mathrm{E}\|)=\mathrm{e}^{-\left(\| \mathrm{E}[\mid / / \mathrm{k})^{2}\right.}
$$

Where $\mathrm{k}$ is a positive constant which is fixed. Airlight map can be estimated iteratively as follows:

$$
A^{t+1}=A^{t}+\lambda\left[\alpha \nabla A^{t}\right]
$$

Where $\lambda(0>\lambda>1)$ is a smoothing parameter. According to the Perona-Malik discrete version of above equation can be written equation (52):

$A(x, y, t+1)=A(x, y, t)+\lambda\left[\alpha_{N}(x, y, t) \nabla_{N} A(x, y, t)+\alpha_{S}(x, y, t) \nabla_{S} A(x, y, t)\right]+$

$\alpha_{E}(x, y, t) \nabla_{E} A(x, y, t)+\alpha_{W}(x, y, t) \nabla_{W} A(x, y, t)$

Where N, S, E and W are North, South, East and West respectively. Symbol $\nabla$ indicates nearest-neighbour differences.

2.7.3. Restoration: For RGB model, intensity is calculated as follows:

$$
\mathrm{I}_{0}(\mathrm{x}, \mathrm{y}, \mathrm{c})=\frac{(\mathrm{I}(\mathrm{x}, \mathrm{y}, \mathrm{c})-\mathrm{A}(\mathrm{x}, \mathrm{y}))}{\ldots}
$$

For HSI model [17] intensity is calculated by same method discussed in RGB model and saturated pixel can be restored as shown in equation (54):

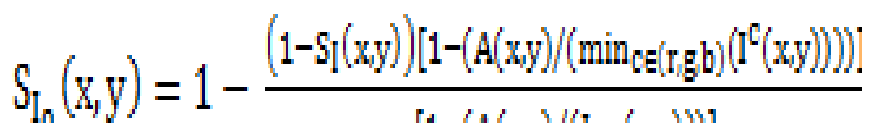

2.7.4. Post Processing: In this step contrast enhancement has done by various methods which are suitable such as histogram equalization histogram stretching, etc.

\subsection{Tarel Method:}

This method has high processing speed. It is the best method used in in-vehicle camera. It is also advantageous to apply on color images as well as on grey images. This algorithm [18] assumes only small objects can have colors with low saturation and is controlled only by a 
few parameters i.e., atmospheric veil inference, image restoration and smoothing, tone mapping.

The mathematical model of foggy image is shown in equation (55):

$$
\mathrm{I}(\mathrm{x}, \mathrm{y})=\mathrm{R}(\mathrm{x}, \mathrm{y})\left(1-\frac{\mathrm{v}(\mathrm{x}, \mathrm{y})}{\mathrm{I}_{\mathrm{g}}}\right)+\mathrm{V}(\mathrm{x}, \mathrm{y})
$$

Where:

$\mathrm{I}(\mathrm{x}, \mathrm{y})$ : foggy image

$\mathrm{R}(\mathrm{x}, \mathrm{y})$ : Defoggy image

$\mathrm{V}(\mathrm{x}, \mathrm{y})$ : Atmospheric veil

$\mathrm{I}_{\mathrm{s}} \quad$ : Sky intensity which is taken as 1

2.8.1. Atmospheric Veil Inference: First of all take the minimum intensity value of RGB plane as $\mathrm{W}(\mathrm{x}, \mathrm{y})$ for each pixel as shown in equation (56) :

$$
\mathrm{W}(\mathrm{x}, \mathrm{y})=\min (\mathrm{I}(\mathrm{x}, \mathrm{y}, \mathrm{t}))
$$

Now, take the median of a $\mathrm{W}$ as in (57):

$$
\mathrm{A}(\mathrm{x}, \mathrm{y})=\operatorname{median}(\mathrm{W}(\mathrm{x}, \mathrm{y}))
$$

Then the local standard deviation (W) is subtracted from A to get B as shown in equation (58)

$$
\mathrm{B}(\mathrm{x}, \mathrm{y})=\mathrm{A}(\mathrm{x}, \mathrm{y})-\operatorname{median}(|\mathrm{W}(\mathrm{x}, \mathrm{y})-\mathrm{A}(\mathrm{x}, \mathrm{y})|)
$$

In the last step, multiply B with a parameter $\mathrm{p}[0,1]$ for good visibility restoration and then finally atmospheric veil is created as given in equation (59):

$$
\mathrm{V}(\mathrm{x}, \mathrm{y})=\max (\min (\mathrm{pB}(\mathrm{x}, \mathrm{y}), \mathrm{W}(\mathrm{x}, \mathrm{y})), 0)
$$

Where $\mathrm{p}=0.95$ and $\mathrm{sv}=41$ (square block window size)

2.8.2. Corner Preserving Smoothening: Previous filter for estimation of A may induce artefacts for large values of sv on very structured scenes such as cities, buildings. Thus an introduction of original filter on previous filter named 'Median of Median along Lines' which is able to preserve edges as well as corners (with obtuse angle).

Take a set of $n_{v}$ centered line segments $S_{i}, 1 \leq i \leq n_{v}$ of uniformly sampled orientations is given. Each segment is of length $S_{v}$. For each pixel and for each segment Si centered on the current pixel, the median value of the intensities along $S_{i}$ is computed and saved as $m_{i}$. When the current pixel is close to an edge, all $\mathrm{m}_{\mathrm{i}}$ are close to the average intensity (I) of the current pixel region.

When the current pixel is close to a corner with angle $\theta$, the percentage of values mi not close to I and then make it equals $1-|\theta| / \pi$; then the median of the mi is close to I. As a result the median of median along lines filter preserves edges as well as corners (with an obtuse angle).

2.8.3. Image Restoration: The equation to restore the image is given below:

$$
\mathrm{R}(\mathrm{x}, \mathrm{y})=\frac{\mathrm{I}(\mathrm{x}, \mathrm{y})-\mathrm{V}(\mathrm{x}, \mathrm{y})}{1-\frac{\mathrm{V}(\mathrm{g}, \mathrm{y})}{\mathrm{I}_{\mathrm{g}}}}
$$


2.8.4. Smoothing Adapted to Contrast Magnification: To reduce the noise and artefacts which is introduced in the image at a time of image compression, a local smoothing is required. This local smoothing must be adapted to the contrast magnification factor $\Upsilon$. Standard deviation $\sigma$ of noise becomes $\gamma \sigma$ after image restoration. By averaging on a window of size $s \times s$, the standard deviation becomes $1 / \sqrt{ }\left(s^{\wedge} 2\right)$ y $\sigma$. Hence, to come back to a noise of standard deviation $\sigma$, s must be equal to the contrast factor $\gamma$. Thus for the locally adapted smoothing to perform a median filter with a square window of size $\mathrm{s} \times \mathrm{s}$ where $\mathrm{s}$ equals the integer part of the contrast factor $\gamma$ is selected. This rule to set $s$ would create over-sized window in very foggy areas. Therefore, an extra parameter si which sets the maximum size of the adapted window. When $s i=1$, this indicates that the effect of the adapted smoothing is cancelled. $\gamma$ is shown in equation (61):

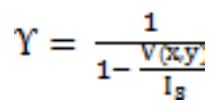

2.8.5. Dedicated Tone Mapping: It is usually done to make the restored image similar to the original image. For that purpose mean and standard deviation of logarithmic image has taken place as shown in equation (62) and (63):

$$
\begin{aligned}
& U(x, y)=R(x, y)^{\frac{a_{I}}{d_{R}} e^{a_{I}-a_{R}} \frac{a_{I}}{d_{R}}} \\
& T(x, y)=\frac{U(x, y)}{1+\left(\frac{1}{255}-\frac{1}{M_{G}}\right) G(x, y)}
\end{aligned}
$$

where

$$
\begin{aligned}
& a_{I}: \text { mean of } \log (I(x, y)) \\
& d_{I}: \text { std of } \log (I(x, y)) \\
& a_{R}: \text { mean of } \log (R(x, y)) \\
& d_{R}: \text { std of } \log (R(x, y))
\end{aligned}
$$

$\mathrm{G}(\mathrm{x}, \mathrm{y})$ : grey level of $\mathrm{U}(\mathrm{x}, \mathrm{y})$

$\mathrm{M}_{\mathrm{G}}$ : Maximum of $\mathrm{G}$

T: Final obtained image

\section{Simulation Setup Parameters}

To evaluate the performance of various defogging techniques, simulation is done in MATLAB. Various common simulation setup parameters are for all techniques are given in Table 1. The simulation parameters specific to techniques are given in Table 2 to 9.

Table 1. Setup Parameters

\begin{tabular}{|l|l|}
\hline Tool Used & Value \\
\hline SOFTWARE USED & MATLAB 2009 \\
\hline SYSTEM RAM & 4 G.B. \\
\hline SYSTEM PROCESSOR & Intel(R)Core(TM)2 Duo \\
\hline CPU & T6500 @ 2.10GHz \\
\hline
\end{tabular}


Table 2. DCP Algorithm Parameters

\begin{tabular}{|l|l|l|}
\hline Symbol & Parameter & Value \\
\hline$\beta$ & Extinction coefficient & 0.5 \\
\hline $\mathrm{W}$ & constant parameter for keep haze away & 0.95 \\
\hline$\lambda$ & weight in data term & $10^{-4}$ \\
\hline
\end{tabular}

Table 3. Adaptive Defogging Algorithm Parameters

\begin{tabular}{|l|l|l|}
\hline Symbol & Parameter & Value \\
\hline GD & Constant Parameter & 0.95 \\
\hline D & Scene Depth & 1.5 \\
\hline$\beta$ & Atmospheric Scattering Coefficient & 0.5 \\
\hline- & Patch Size Used & $15^{*} 15$ \\
\hline
\end{tabular}

Table 4. Anisotropic Diffusion Algorithm Parameters

\begin{tabular}{|l|l|l|}
\hline Symbol & Parameter & Value \\
\hline$\beta$ & Atmospheric Light Estimation Coefficient & 0.95 \\
\hline $\mathrm{A}$ & Conduction Coefficient & $0 / 1$ \\
\hline $\mathrm{K}$ & Constant Used In Diffusion Process & 20 \\
\hline$\lambda$ & Smoothness Parameter & {$[0,1]$} \\
\hline
\end{tabular}

Table 5. Tarel Algorithm Parameters

\begin{tabular}{|l|l|l|}
\hline Symbol & Parameter & Value \\
\hline $\mathrm{P}$ & Strength Controlling Visibility Factor & 0.95 \\
\hline $\mathrm{S}_{\mathrm{v}}$ & Window Size & 41 \\
\hline
\end{tabular}

Table 6. DCP Algorithm Parameters

\begin{tabular}{|l|l|l|}
\hline Symbol & Parameter & Value \\
\hline $\mathrm{G}$ & Constant & 0.95 \\
\hline $\mathrm{D}$ & Scene depth & 1.5 \\
\hline $\mathrm{B}$ & Atmospheric Scattering Coefficient & 0.5 \\
\hline $\mathrm{t}_{0}$ & Minimum Transmission Limit & 0.1 \\
\hline- & Patch Size Used & $15^{*} 15$ \\
\hline
\end{tabular}

Table 7. IDCP Algorithm Parameters

\begin{tabular}{|l|l|l|}
\hline Symbol & Parameter & Value \\
\hline $\mathrm{D}$ & Scene Depth & 1.5 \\
\hline $\mathrm{B}$ & Atmospheric Scattering Coefficient & 0.5 \\
\hline $\mathrm{t}_{0}$ & Minimum Transmission Limit & 0.35 \\
\hline- & Patch Size Used & $31 * 31$ \\
\hline
\end{tabular}


Table 8. IDCP Using Guided Filter Algorithm Parameters

\begin{tabular}{|l|l|l|}
\hline Symbol & Parameter & Value \\
\hline$\alpha$ & Constant & 2 \\
\hline$G D$ & Constant & 0.95 \\
\hline$D$ & Scene depth & 1.5 \\
\hline$B$ & Atmospheric Scattering Coefficient & 0.5 \\
\hline$t_{0}$ & Minimum Transmission Limit & 0.35 \\
\hline- & Patch Size Used & $15^{*} 15$ \\
\hline
\end{tabular}

Table 9. Histogram Specification Algorithm Parameters

\begin{tabular}{|l|l|l|}
\hline Symbol & Parameter & Value \\
\hline$G D$ & Constant Parameter & 0.95 \\
\hline$D$ & Scene Depth & 1.5 \\
\hline B & Atmospheric Scattering Coefficient & 0.5 \\
\hline$t_{0}$ & Minimum Transmission Limit & 0.1 \\
\hline- & Patch Size Used & $15^{*} 15$ \\
\hline
\end{tabular}

\section{Performance Metrics:}

\subsection{Mean Square Error (MSE):}

The MSE represents the cumulative squared error between the compressed and the original image. The lower the value of MSE, the lower the error. Given a noise-free $m \times n$ monochrome image I and its noisy approximation K, MSE is defined in equation (64):

$$
\operatorname{MSE}=\frac{1}{2} \sum_{i=n}^{m-1} \sum_{i=n}^{n-1}[I(i, j)-K(i, j)]^{2}
$$

\subsection{Peak Signal to Noise Ratio (PSNR):}

It computes the peak signal-to-noise ratio, in decibels, between two images. This ratio is often used as a quality measurement between the original and a compressed image. The higher the PSNR, the better the quality of the compressed or reconstructed image as shown in equation (65).

$$
\begin{aligned}
& \text { PSNR }=10 \cdot \log _{10}\left(\frac{\mathrm{MAX}_{\mathrm{I}}^{2}}{\mathrm{MSE}}\right) \\
& \quad=20 \cdot \log _{10}\left(\frac{\mathrm{MAX}_{\mathrm{I}}}{\sqrt{\mathrm{MSE}}}\right) \\
& =20 \cdot \log _{10}\left(\mathrm{MAX}_{\mathrm{I}}\right)-10 \cdot \log _{10}(\mathrm{MSE})
\end{aligned}
$$

MAX is the max intensity value in an image i.e. 1 in double.

\subsection{Mean Absolute Error (MAE):}

It measures network performance as the mean of absolute errors. 


\subsection{Normalized Colour Difference (NCD):}

It is used to measure the degradation in colour quality in colour images.

\subsection{Computation Time:}

It is the time taken by algorithm to remove fog.

\subsection{Histogram:}

It is a graph indicating the number of times each grey level occurs in the image.

\section{Results}

Figure 10. shows snapshots of original image after applying defogging images.

\subsection{Snapshots of Image}

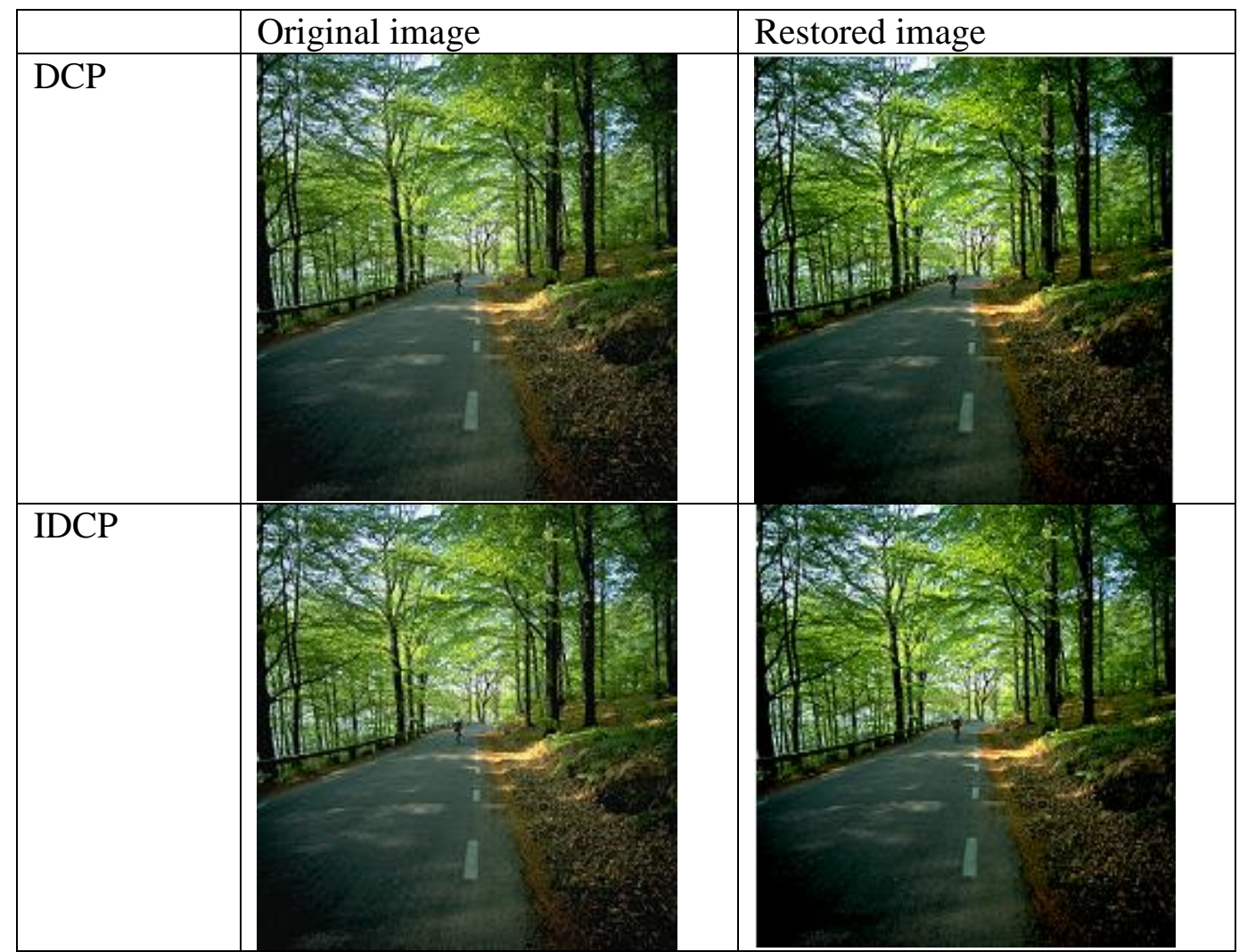




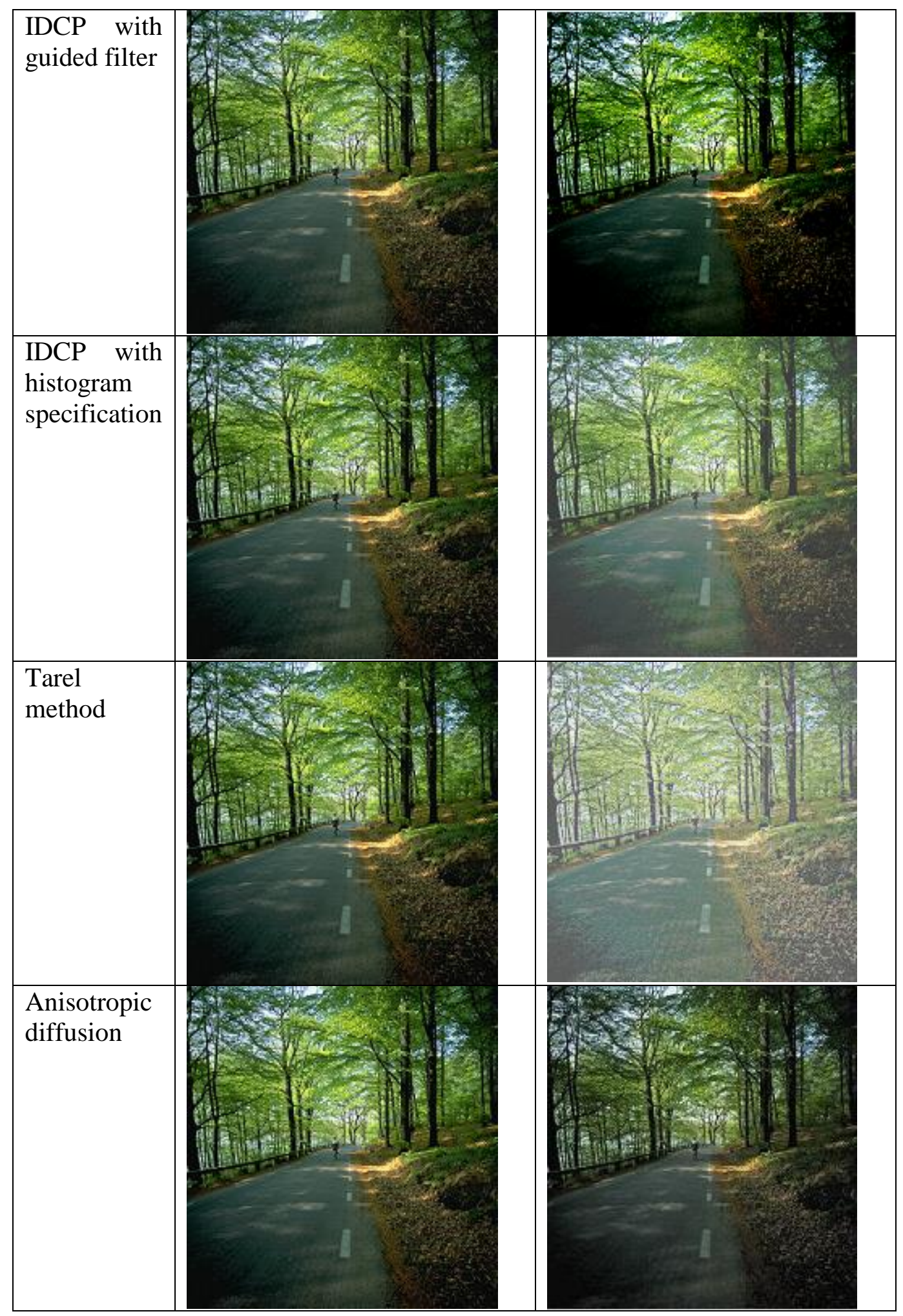




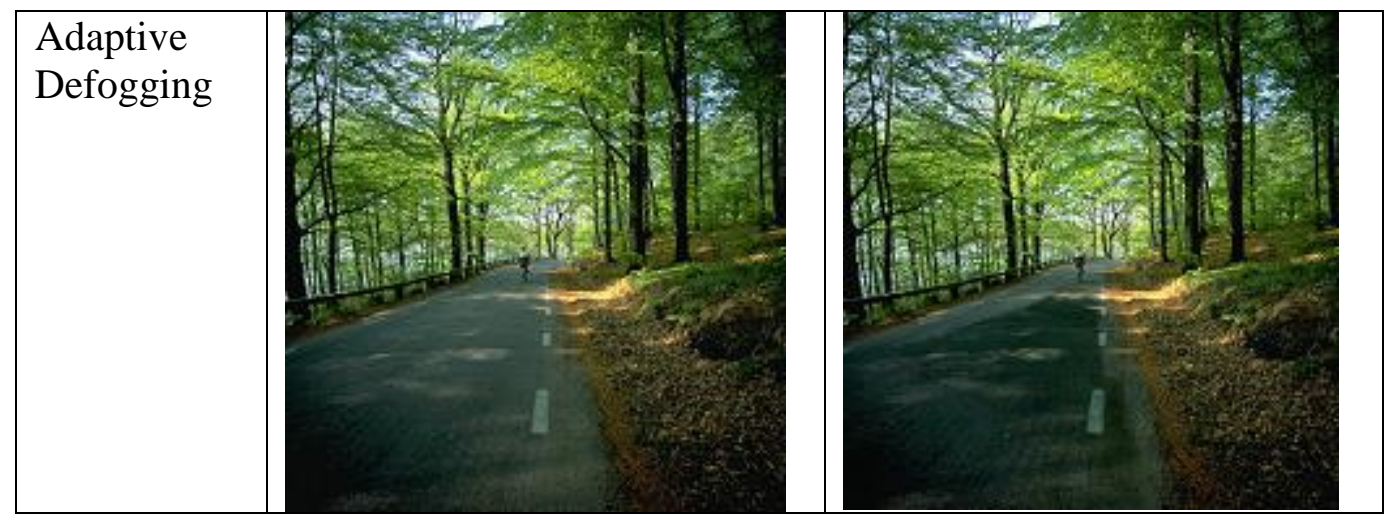

Figure 10. Snapshots Various of Defogging Techniques Discussed Above

\subsection{Results of MAE}

Figure 11(a) shows the results of MSE. The following inference can be drawn:

1. Adaptive Defogging and Anisotropic diffusion gives the best results for MAE as they are lower than other techniques.

2. Histogram Specification has highest MAE value.

3. As the size of image increases, values of MAE generally increase, except in histogram specification and anisotropic diffusion method where the values decrease.

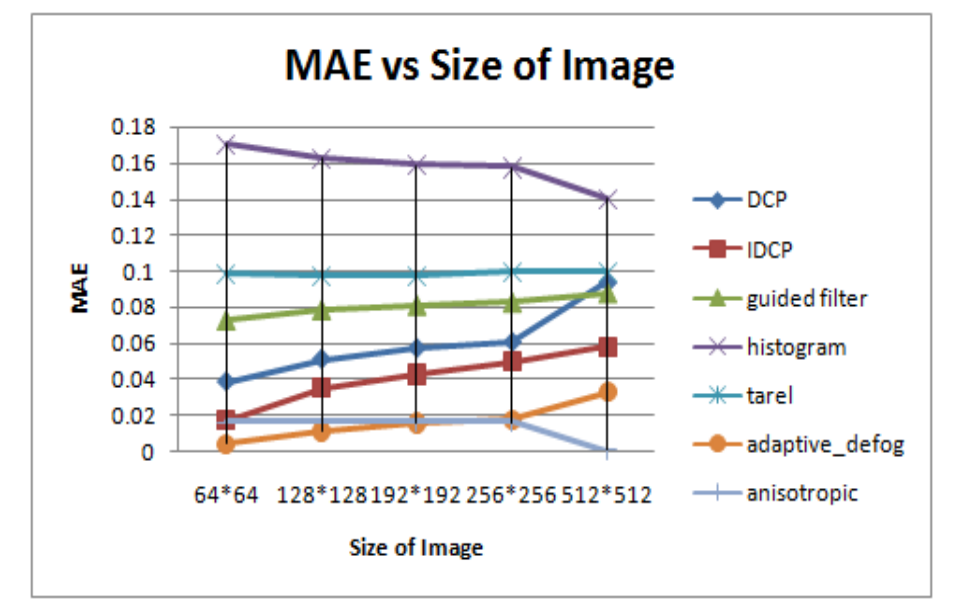

Figure 11(a). Graph between MAE and Size of Image

\subsection{Results of MSE and PSNR}

Figure 11(b) and 11(c) shows MSE and PSNR results of defogging strategies: 


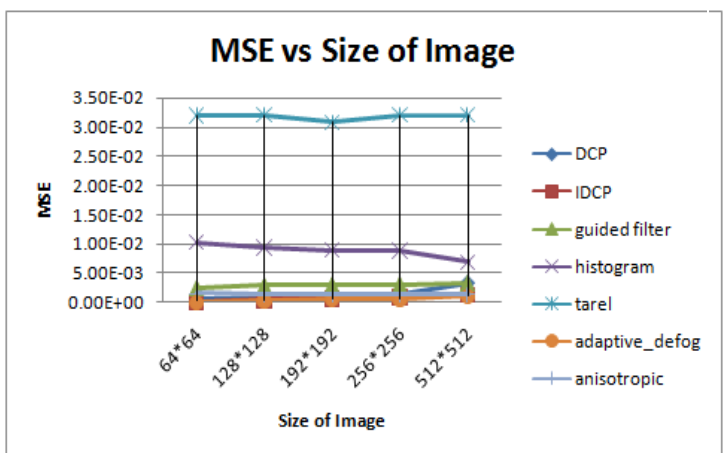

Figure 11(b). Graph Between MSE and Size of Image

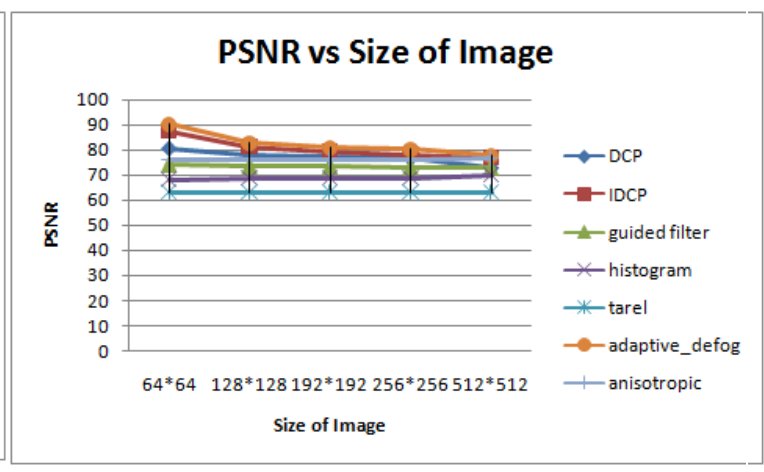

Figure 11(c). Graph Between PSNR and Size of Image

Figure 11(b) and 11(c) shows the MSE results for varying image sizes. Following inference can be drawn from the results:

- As the image size increases the MSE decreases, PSNR increases for all techniques except Adaptive Defogging technique.

- Adaptive defogging and IDCP technique shows the best results in terms of PSNR.

\subsection{Results of NCD:}

Figure 11(d) shows the results of NCD for varying image sizes. The following inference can be drawn:

- As the image size increases, the value of $\mathrm{NCD}$ decreases in case of histogram specification.

- Adaptive Defogging and IDCP techniques have lower NCD values than any other technique.

- DCP has the highest NCD values.

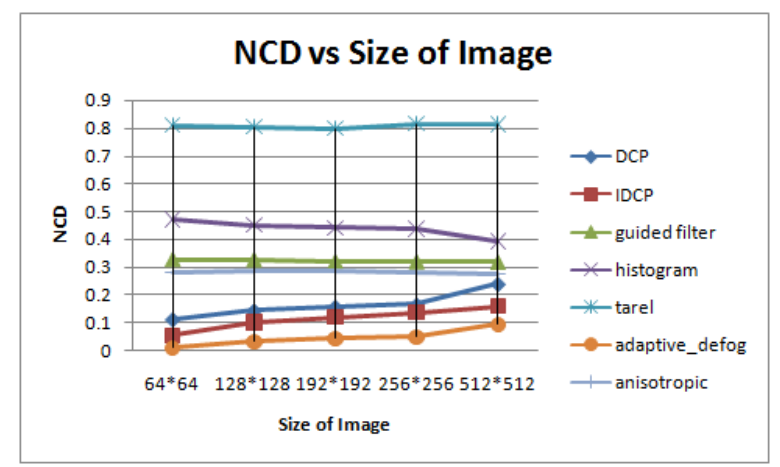

Figure 11(d). Graphs Showing NCD vs Size of Image

\subsection{Results of Time Complexity:}

The following inference can be drawn from Figure 11(e):

- As the image size increases, time complexity also increases for all the techniques. 
- Anisotropic Diffusion and Tarel methods have lowest time complexity but affect the picture quality.

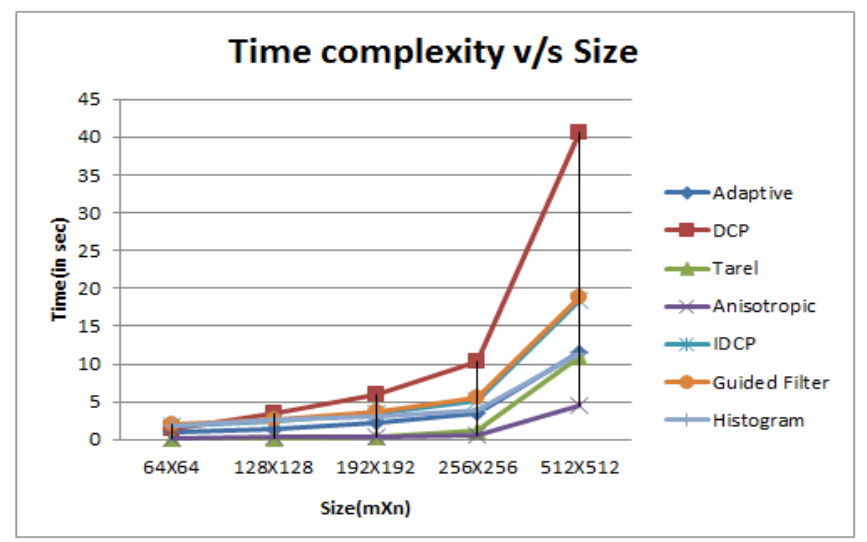

Figure 11(e). Graphs Showing Time Complexity vs Size of Image

\section{Conclusion}

The overall comparison of all the techniques is given in Table 10 .

Table 10. Overall Comparison

\begin{tabular}{|l|l|l|l|l|l|l|l|}
\hline Parameter & DCP & IDCP & $\begin{array}{l}\text { Guided } \\
\text { Filter }\end{array}$ & Histogram & $\begin{array}{l}\text { Tarel } \\
\text { Method }\end{array}$ & $\begin{array}{l}\text { Anisotropic } \\
\text { Diffusion }\end{array}$ & $\begin{array}{l}\text { Adaptive } \\
\text { Defogging }\end{array}$ \\
\hline MSE & 3 & 2 & 5 & 6 & 7 & 4 & 1 \\
\hline PSNR & 3 & 2 & 5 & 6 & 7 & 4 & 1 \\
\hline NCD & 3 & 2 & 5 & 6 & 7 & 4 & 1 \\
\hline MAE & 4 & 3 & 5 & 7 & 6 & 1 & 2 \\
\hline Time & 4 & 5 & 6 & 7 & 2 & 1 & 3 \\
\hline
\end{tabular}

Where:

1: BEST

2: BETTER

3: GOOD

4: SATISFACTORY

5: BAD

6: POOR

7: WORST

Looking at the observations, following inference may be made:

- IDCP and Adaptive defogging techniques have much high PSNR and lower MSE in comparison to other techniques.

- NCD and MAE are also lower in case of IDCP and Adaptive defogging techniques, showing good results. 
- The time complexity of Tarel and Anisotropic Diffusion method is very low, but is affecting the PSNR. Hence, to increase PSNR, time complexity is increased to some extent.

\section{References}

[1] S. G. Narasimhan and S. K. Nayar, "Vision and the Atmosphere", International Journal on Computer Vision, vol. 48, (2002), pp. 233-254.

[2] S. G. Narasimhan and S. K. Nayar, "Chromatic Framework for Vision in Bad Weather", Proceedings of IEEE Conference on Computer Vision and Pattern Recognition, vol. 1, (2000) June, pp. 598-605.

[3] R. Fattal, "Single Image Dehazing", Proceedings of ACM SIGGRAPH, (2008), pp. 1-9.

[4] R. Tan, "Visibility in Bad Weather from a Single Image", Proceedings of IEEE Conference on Computer Vision and Pattern Recognition, (2008) June, pp. 1-8.

[5] Y. Y. Schechner, S. G. Narasimahan and S. Nayar, "Instant Dehazing of Images Using Polarization", Proc. IEEE Conf. Computer Vision and Pattern Recognition, vol. 1, (2001), pp. 325-332.

[6] Y. Y. Schechner, S. G. Narasimahan and S. Nayar, "Polarization-based vision through haze", Applied Optics, vol. 42, no. 3, (2003), pp. 511-525.

[7] K. He, J. Sun and X. Tang, "Single image haze removal using dark channel prior", IEEE Int. Conf. On Computer Vision and Pattern Recognition, (2009), pp. 1956-1963.

[8] J. Oakley and B. Satherley, "Improving image quality in poor visibility conditions using a physical model for constant degradation”, IEEE Transactions on Image Processing, vol. 7, no. 2, (1998), pp. 167-169.

[9] A. Levin, D. Lischinski and Y. Weiss, "A Closed Form Solution to Natural Image Matting", Proceedings of IEEE Conference on Computer Vision and Pattern Recognition, vol. 1, (2006), pp. 61-68.

[10] C. Tomasi and R. Manduchi, "Bilateral Filtering for Grey and Colour Images", Proc. Sixth IEEE Int'1 Conf. Computer Vision, (1998), pp. 839-846.

[11] Yan Wang and Bo Wu, "Improved Single Image Dehazing using Dark Channel Prior", Proc. IEEE Conf. Intelligent Computing and Intelligent Systems (ICIS), vol. 2, (2010), pp. 789-792.

[12] Y. Xiong and H. Yan, "Improved Single Image Dehazing using Dark Channel Prior", Journal of Computational Information Systems, vol. 9, (2013), pp. 5743-5750.

[13] S. Yang, Q. Zhu, J. Wang, D. Wu and Y. Xie, "An Improved Single Image Haze Removal Algorithm Based on Dark Channel Prior and Histogram Specification”, Proc. 3rd International Conf. On Multimedia Technology, Atlantis Press, http://www.springer.com/lncs, (2013), pp. 279-292.

[14] I. Yoon, S. Kim, D. Kim, M. H. Hayes and J. Paik, "Adaptive Defogging with Color Correction in the HSV Color Space for Consumer Surveillance System”, Consumer Electronics, IEEE Transactions, vol. 58, no. 1, (2012), pp. 111-116.

[15] A. K. Tripathi and S. Mukhopadhyay, "Single image fog removal using anisotropic diffusion", IET Image Process, vol. 6, no. 7, (2012), pp. 966-975.

[16] P. Perona and J. Malikm, "Scale space and edge detection using anisotropic diffusion", IEEE Transaction on Pattern Analysis and Machine Intelligence, vol. 12, no. 7, (1990), pp. 629-639.

[17] R. C. Gonzaless and R. E. Woods, "Digital image processing", (Addison-Wesley, Reading, MA, 2nd edn.), (1992).

[18] J. P. Tarel and N. Hautiere, "Fast visibility restoration from a single color or gray level image", IEEE Int. Conf. on Computer Vision, (2009), pp. 2201-2208.

[19] K. He, J. Sun and X. Tang, "Single image haze removal using dark channel prior", IEEE transactions on "pattern analysis and machine intelligence", vol. 33, no. 12, (2011) December, pp. 2341-2353.

[20] M. Chen, A. Men, P. Fan and B. Yang, "Single image defogging", IEEE Conference on Network Infrastructure and Digital Content, (2009) November, pp. 675-679.

\section{Authors}

Atul Gujral, received B.Tech. degree from the Career Institute of Technology and Management, Faridabad, India in 2012. He is currently working toward the M. Tech. degree form YMCAUST, Faridabad, India. His interests include digital signal processing.

Aditi, received B.Tech. degree from Banasthali University main campus,Newai,Tonk, India in 2012. He is currently working toward the M. Tech. degree form YMCAUST, Faridabad, India. Her interests include digital signal processing. 
International Journal of Signal Processing, Image Processing and Pattern Recognition

Vol.7, No.3 (2014)

Shailender Gupta, received B.Tech, M.Tech and Ph.D from YMCA University of Science and Technology, Faridabad, India. Currently he is working as Assistant Professor in Electronics Engineering Department in YMCAUST. His interests include digital signal processing and Mobile Ad hoc Network.

Bharat Bhushan, received B.Tech from Punjab Engineering College, Chandigarh, India and M.Tech from YMCA University of Science and Technology, Faridabad, India. Currently he is working as Assistant Professor in Electronics Engineering Department in YMCAUST. His interests include digital signal processing. 